\title{
ДВА БЫСТРЫХ АЛГОРИТМА ПРОЕКТИРОВАНИЯ ТОЧКИ НА СТАНДАРТНЫЙ СИМПЛЕКС ${ }^{1}$
}

\author{
В.Н. Малоземов, Г.Ш. Тамасян
}

Санкт-Петербургский государственный университет, Санкт-Петербург e-mail:malv@math.spbu.ru,g.tamasyan@spbu.ru

В докладе рассматриваются два быстрых алгоритма проектирования точки $c \in \mathbb{R}^{n}$ на стандартный симплекс $\Lambda \subset \mathbb{R}^{n}$, определяемый условиями

$$
\sum_{i=1}^{n} x_{i}=1 ; \quad x_{i} \geqslant 0, i \in 1: n .
$$

Исследуемая задача ставится следующим образом:

$$
\frac{1}{2} \sum_{i=1}^{n}\left(x_{i}-c_{i}\right)^{2} \rightarrow \min _{x \in \Lambda}
$$

где $c_{1}, \ldots, c_{n}$ - координаты проектируемой точки $c$. Решение этой задачи существует и единственно. Обозначим его $x^{*}$.

В работе [1] был описан быстрый алгоритм нахождения $x^{*}$. Идея алгоритма основана на алгебраическом анализе условий оптимальности в форме Куна-Таккера для задачи (1).

Ранее, появилась работа [2], в которой также предлагался конечный алгоритм решения задачи (1). Этот алгоритм имеет геометрический характер, что подтверждается в недавней работе [3].

В данном докладе мы даем усовершенствованный вариант описания и обоснования алгоритма из [2] и приводим результаты численных экспериментов по сравнению двух быстрых алгоритмов решения задачи (1). Отметим одну интересную особенность (см. [4]): когда один из двух алгоритмов проектирования имеет максимальную трудоемкость, у второго алгоритма трудоемкость минимальна.

\section{ЛИТЕРАТУРА}

1. Малоземов В. Н., Певный А. Б. Бъстрый алгоритм проектирования точки на симплекс // Вестник СПбГУ. Сер. 1. 1992. Вып. 1 (№ 1). С. 112-113.

2. Michelot C. A finite algorithm for finding the projection of a point onto the canonical simplex of $\mathbb{R}^{n} / /$ JOTA. 1986. Vol. 50. No 1. P. 195-200.

3. Causa A., Raciti F. A purely geometric approach to the problem of computing the projection of a point on a simplex //JOTA. 2013. Vol. 156. No 2. P. 524-528.

4. Малоземов В. Н., Тамасян Г. Ш. Ещё один быстрый алгоритм проектирование точки на стандартный симплекс // Семинар «DHA \& CAGD». Избранные доклады. 5 сентября 2013 г. (http://dha.spb.ru/reps13.shtml\#0905)

\footnotetext{
${ }^{1}$ Работа выполнена при финансовой поддержке РФФИ (проекты 12-01-00752_a, 14-01-31521_мол_а)
} 